\title{
Low-voltage grid upgrades enabling islanding operation
} \author{
, Ricardo Santos 6 , José Gouveia ${ }^{4}$ \\ ${ }^{1}$ Centre for new energy of technologies, EDP NEW R\&D, Portugal \\ ${ }^{2}$ Corporate technology, SIEMENS AG, Germany \\ ${ }^{3} R \& D$ department, GPTECH, Spain \\ ${ }^{4}$ Centre for power and energy systems, INESC-TEC, Portugal \\ ${ }^{5}$ Energy Management, SIEMENS SA, Portugal \\ ${ }^{6}$ Innovation and development, EDP Distribuição, Portugal \\ $凶$ E-mail: ricardo.andre@edp.pt
}

Ricardo André1 ${ }^{凶}$, Filipe Guerra ${ }^{1}$, Matthias Gerlich ${ }^{2}$, Michael Metzger $^{2}$, Salvador Rodriguez ${ }^{3}$, Clara Gouveia ${ }^{4}$, Carlos Moreira ${ }^{4}$, José Damásio $^{5}$

\begin{abstract}
This article addresses the developments ongoing in SENSIBLE, an H2020 funded project focused on energy storage and energy management, which demonstration occurs in Évora-Portugal, Nottingham, UK and Nuremberg, Germany. Currently, the presented work focuses on the developments necessary to make possible islanding operation in low-voltage grids, where new assets like storage and new automation and protection schemes are necessary to guaranty safety and reliability of low-voltage grids. These developments are a result of the ongoing works under one of SENSIBLE use cases which demonstrations occurs in a small village in Évora district in Portugal.
\end{abstract}

\section{Introduction}

Over last decades, due to environmental and sustainability policies, distributed energy resources (DER) have been deployed along distribution grids. Since DER are available, islanding operation may become an important resource to the distribution system operator (DSO), mainly in rural overhead grids (exposed to weather) with no redundancy. If any technical constraint in upstream grid occurs and DER are available, DSO could comply with its main obligation, ensuring continuity of supply and resilience of the grid. Nevertheless, DER volatility and unpredictability must be balanced with electrical 'flexibility' which can be provided either by demand-side management (DSM) schemes or even energy storage systems (ESS) which can bring additional advanced functionalities. The integration of storage as well as the increased flexibility of loads and generation, complemented by adequate control and coordinated management, enables the islanded operation of the low-voltage (LV) grids. One of SENSIBLE [H2020-SENSIBLE: Storage-Enabled Sustainable Energy for Buildings and Communities, Grant no. 645963.] use cases will explore the possibility of operating a LV grid and evaluate the necessary changes to the existing infrastructure to allow it.

In Portugal, a standard LV grid secondary substation (SS) has a topology similar as outlined in next section (Fig. 1) where two main aspects shall be considered: (i) standard SS are designed to be simple, cheap, reliable and robust; (ii) features like islanding operation and storage functionalities have not been considered until now, since technology was not available at an affordable cost.

Moreover, energy-storage applications in LV grids have not yet been tested in Portugal as new and powerful asset to be managed by the DSO or as a service provided by a third party. This paper describes the solutions that are being developed under SENSIBLE project to enable islanding operation and energy storage management.

\section{Microgrid requirements and demonstrator overview}

In terms of hardware, as outlined in Fig. 1, SS basically consists in a medium-voltage (MV) panel composed by switches/disconnectors, an $\mathrm{MV} / \mathrm{LV}$ power transformer, LV feeders and public lightning circuit protected by fuses. Regarding the monitoring capabilities, the SS includes energy metering as well as a smart grid element called distribution transformer controller (DTC).

It has some simple automation functionalities and is able to collect smart meters' (SMs) measurement information through automatic meter infrastructure, operated by the DSO. Moreover, DTC is able to manage DSM signals in case of grid technical constraints requesting flexibility from customers.

\section{Microgrid requirements}

To enable the integration of distributed storage and allow the islanding operation of LV network, the SS must be upgraded at four different layers as follows.

\subsection{Network operation and control}

The autonomous operation of the LV network requires the implementation of specific tools and control to ensure the stability of the island. In addition to the local control strategies implemented at the storage inverter level (ensuring frequency and voltage regulation), it is necessary to monitor and manage the islanded system. In this sense, the operation and control will be implemented both at central systems at the SS at four levels.

High-level tools, included in distribution management system (DMS), design to monitor and manage the reserve capacity of the system provided by the storage units and flexible loads controlled through DSM. 


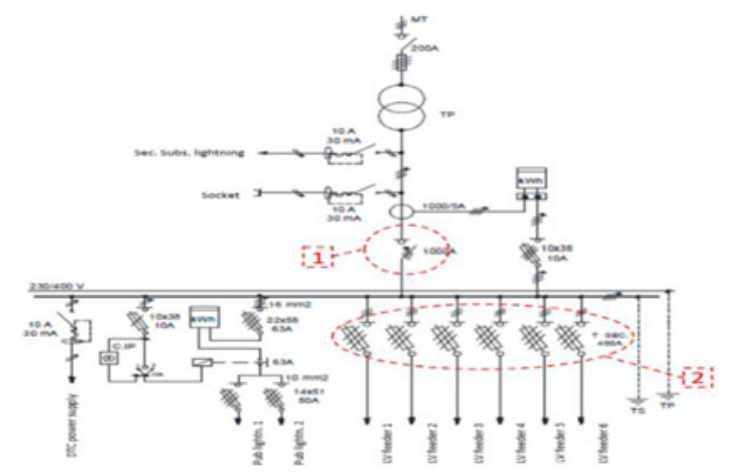

Fig. 1 Standard LV secondary substation architecture in Portugal [1]

At the SS, the DTC need to be upgraded to: (i) operate and monitor ESS installed in LV grid; (ii) transmit information to upstream DMS; (iii) implement a local automation and operation philosophy in case of islanding. The DTC will also be supported by the islanding manager (IM) which ensures the coordination between storage devices and ensures secondary control.

\subsection{Automation, control and protection upgrades}

It is necessary to replace the LV switchgear switch/disconnector by a circuit breaker to be able to isolate from grid in case of fault and synchronise when grid is available (i). It is also necessary to replace the feeders' fuse-switch by LV circuit breaker (ii) to ensure selectivity in case of islanding due to low short-circuit currents provided by ESS power electronics [2]

A protection relay to enabling islanding must ensure safety for grid and clients, which coordination and selectivity studies in islanding mode were also carried out [2].

\subsection{Energy storage assets integration}

Electrochemical storage ( $\mathrm{Li}$-ion batteries) was installed to provide flexibility in islanding operation, both at SS level and at feeder level. At SS level, the ESS will be operated with V/f control and at feeder level as a P/Q control. Drop control strategies will be implemented at ESS level.

ESS batteries were sized so that an islanding could be possible in $80 \%$ of outrages/failures in MV grid side. In terms of energy, $30 \mathrm{~min}$ islanding duration would cover also $95 \%$ of the outages, based on historical data [3]. This results in a necessary capacity of approximately $80 \mathrm{~kW}$ and energy of at least $40 \mathrm{kWh}$.

An electromechanically storage device (flywheel $-125 \mathrm{~kW}$ ) will be installed to provide power in complement to batteries in case of transient regimens providing short-time peak active power.

\subsection{SMs and smart homes improvements}

SMs able to provide DSM schemes than can request additional flexibility from clients, based on a residential home energy management system able to manage residential storage, residential PV generation and residential flexible loads, in case of any technical constraint in distribution grid [4]. This flexibility will be requested my high-level tools in case of urgent constraints.

\section{Demonstrator overview}

Évora demonstrator is outlined in Fig. 2, including two SS as well as an MV client on right side. Just one of SS will be equipped to work in islanding, namely with the installation of the flywheel, battery storage and advanced. Nevertheless, an additional ESS are installed in the other SS for exploring the EES control for the improvement of network operation conditions (Fig. 3). ESS is also

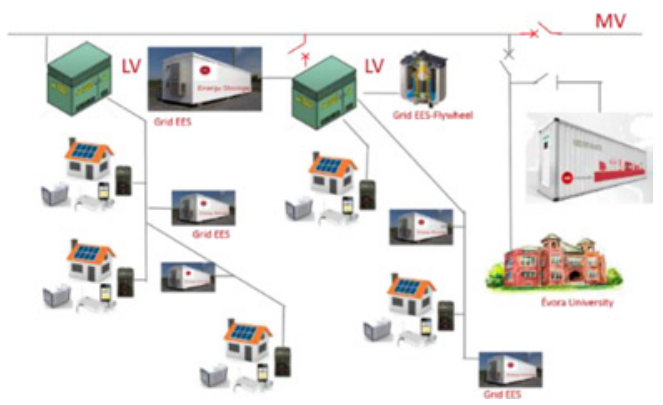

Fig. 2 Évora demonstrator architecture

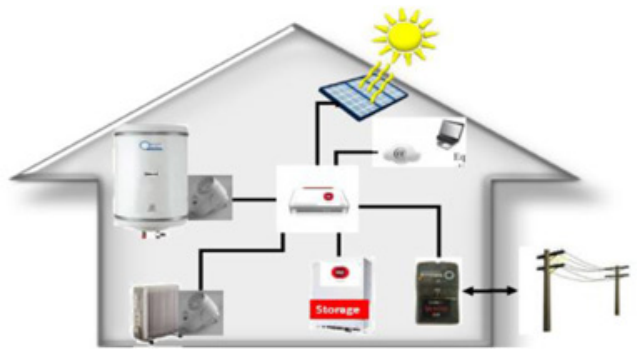

Fig. 3 Residential energy management equipment in SENSIBLE's Evora demonstrator [4]

installed at MV level, but its application and use case is out of scope of this article.

In Évora demonstrator, $10 \%$ of 240 its clients will be able to provide flexibility to the DSO operation in case of technical constraints (Fig. 4). This residential flexibility is activated by DSO through each SM and managed locally by the HEMS system, which will control the PV, residential storage and controllable loads like water heaters to change its power flow with the LV network [4].

Fig. 5 outlines the final location of SS and EES devices where a $50 \mathrm{kVA} / 44 \mathrm{kWh}$ at SS level and a $30 \mathrm{kVA} / 22 \mathrm{kWh}$ will be installed at a feeder level. The total capacity of $80 \mathrm{~kW} / 66 \mathrm{kWh}$ was achieved to fulfil the minimum capacity that allows an islanding to occur with up to $90 \%$ of reliability considering that SS load profile and MV feeder fault historical data. In terms of energy, the ESS may be a bit oversized but the ratio power/capacity which results from in Li-ion must be respected.

The flywheel will also be installed at SS level with a capacity of $125 \mathrm{~kW} / 0.9 \mathrm{kWh}$. The reduced energy stored will be used just in transient regimen where a fast response is necessary.

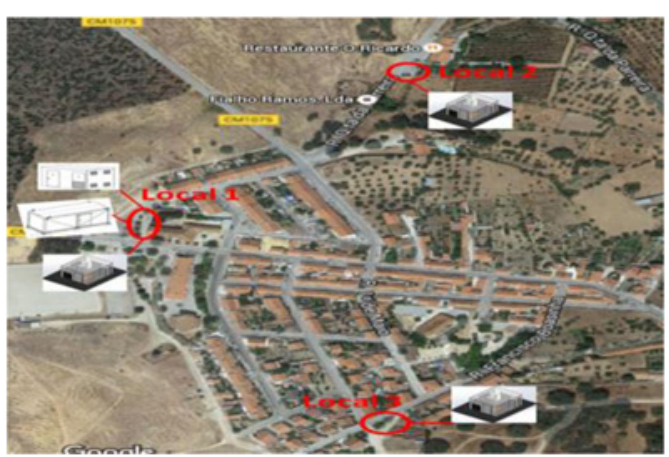

Fig. 4 Évora demonstrator overview [Google] 


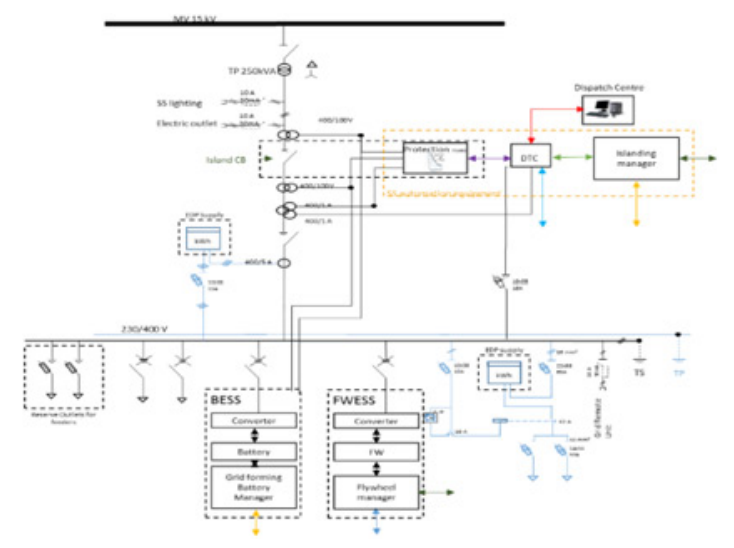

Fig. 5 Standard LV secondary substation architecture in Portugal [EDP Distribuição]

\section{Hardware features}

The DTC will work as a gateway, providing the information collected from the LV grid, namely ESS and protection devices and send it to the central systems. At the same time, it will be responsible for sending the control set-points and signals downstream. More importantly, it will act as the local brain of the SS and islanded system, incorporating automation for the disconnection and reconnection of the LV system to the main grid. It will also be complemented by the IM, which will promote the coordination among ESS, namely optimising power electronics droop functions considering ESS state-of-charge (SoC).

The SS power infrastructure is represented in Fig. 5. The main circuit breaker (CB) will be responsible for the disconnection/ connection to the main grid. In addition, at the LV switchboard will be equipped with a circuit breaker per feeder. LV switches were installed by two reasons: (i) guarantee selectivity in case a fault occurs in a LV feeder when operating in islanding, allowing its isolation without causing a blackout; (ii) since ESS have low short-circuit current when compared with typical MV grid, in case of a failure and under certain circumstances the necessary current to blow the fuses could not be achieved. This is one of the main challenges of operating a system-dominated by power electronics.

Fig. 6 represents the transient of normal operation to islanding where the complementarity between all energy storage sources can be seen. This transient operation is explained in [2] where protection topic is also addressed considering the fault simulations in several fault scenarios.

The protection simulation topic is particularly important in islanding operation where power electronics typically limit short-circuit currents which may be an issue to detect.

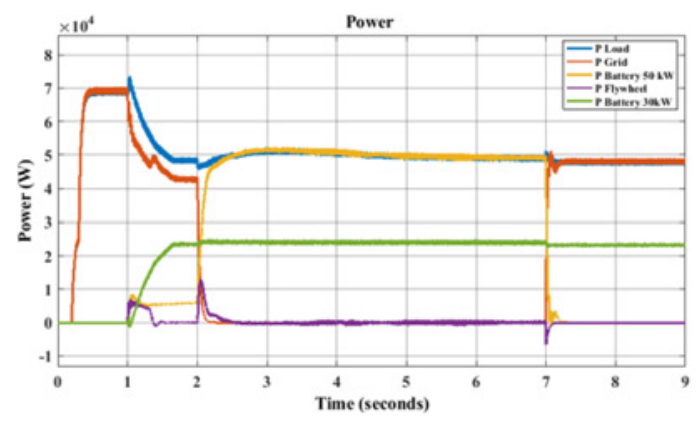

Fig. 6 Active power profile on the grid on transition grid-connected/ islanding operations [2]

\section{Control and tools features}

Flexibility and reliability are key factors in microgrid (MG) due to its ability to operate both interconnected to the main grid or autonomously. However, operating autonomously in a system with high penetration of DER and consequently a low inertia can be quite challenging.

During islanded operation, the MG frequency regulation strategies will try to ensure continuous power balancing with minimum dependence of communication systems. Similarly to the conventional system, the organisation of MG control structures typically follows an hierarchical arrangement comprising primary, secondary and tertiary control. During islanded operation, the MG primary control layer is responsible for frequency and voltage regulation strategies that will assure the continuous power balancing with minimum dependence of communication system.

Primary control is usually based on a droop strategy implemented as an external control loop of the storage unit coupling inverter, controlled as a voltage source inverter (VSI). As represented in Fig. 7, the local power imbalance is supported by VSI and will cause frequency variations in accordance to the active power-frequency (P-f) droop, similarly to the response of the conventional synchronous machines. Then, the secondary control layer will be responsible to restore MG frequency and node voltages to reference set-points. MG secondary control can be performed by the storage devices and/or other controllable microgeneration units connected to the system. In the context of SENSIBLE project, primary control will be provided by Siemens Flywheel and GPTech $80 \mathrm{kVA}$ battery inverter. However, considering the limited energy capacity of the flywheel system only GPTech battery inverter will ensure secondary frequency regulation, by correcting frequency to nominal value while continue to supply the MG loads.

The effectiveness of MG frequency regulation strategies will depend on the power disturbance severity and on the availability of the resources to participate in frequency regulation. Primary control will only ensure power balance if there is sufficient storage capacity and if the inverters' maximum power limits are not surpassed. Similarly, secondary control will fail if the MG lacks sufficient reserve capacity to compensate the power injected by the VSI and correct frequency deviation.

Therefore, local control should be supported by high-level supervisory management functionalities specifically designed to ensure adequate MG controllability during islanding conditions and improve the system resiliency.

In the context of SENSIBLE project, a tool is being developed for providing an effective management of the MG during autonomous to maximise the MG power and energy reserve capacity and the time the LV system can operate autonomously. The algorithm dispatches the storage units participating in the MG frequency control considering their power and energy reserve capacity. Then, if the reserve capacity is insufficient, the algorithm will schedule additional control actions taking advantage of the residential flexibility, managed through the home energy management systems (HEMS).

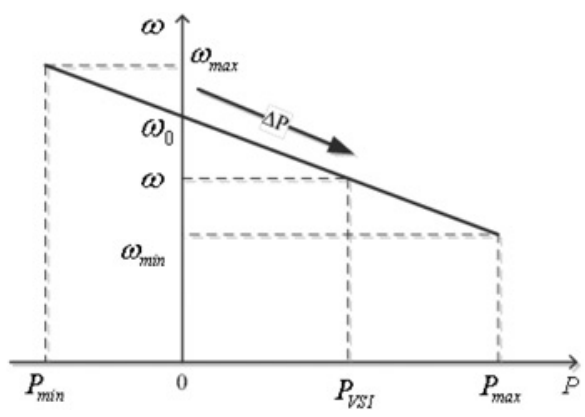

Fig. 7 VSI active power - frequency droop 
The MG emergency balance tool will set the operational plan for the MG devices, namely grid-supporting storage and residential flexible resources, being possible for the DSO to set the algorithm time range $(1 \mathrm{~h}$ or $2 \mathrm{~h})$, considering the time resolution of the real-time and forecasting data (it was considered $15 \mathrm{~min}$ time steps). The developed method considers the daily load and microgeneration forecasts and also the real MG state, namely the capacity of the storage units.

The algorithm will run both during interconnected and islanded operation. During interconnected operation, the algorithm determines an emergency schedule for distributed storage units, considering that an unplanned islanding might occur in the next time-step. The operational plan will be constantly being refreshed according to the real grid data, which will be updated at a periodicity of $15 \mathrm{~min}$. In case the VSI SoC and/or its active power set-point exceeds a pre-defined maximum or minimum threshold limit, it will be necessary to resort to residential flexibility management to prevent the MG blackout.

During islanded operation, the algorithm is supposed to set an operational plan for the specified algorithm time range, from the moment the MG islanding occurs. Nonetheless, considering the potential error of the load and microgeneration forecasts, the deviation between the real and the forecasted load and microgeneration values will be monitored. When the error goes beyond a specified threshold, the algorithm will determine a new solution, updating the forecasted values to the measured ones. The operational plan is supposed to be updated upon the reception of new measured data relatively to the real grid of the storage unit's capacity.

The algorithm can be summarised in three different steps:

(i) Characterise the $M G$ operating state: based on the information collected by the DTC at the SS and remotely from the SM. So, at a defined periodicity, the tool will collect the data relative to the MG storage devices, loads and microgeneration state. Based on the collected data, the algorithm determines the power and energy reserves available.

(ii) Emergency dispatch of $M G$ controllable resources: The algorithm defines the operation plan for the defined timeframe ( 1 or $2 \mathrm{~h}$ ), based on the current state of the network (load, microgeneration and storage devices) and in the load and microgeneration forecasts for the considered time range. The plan will consist in a set of power set-points for a defined time step for the MG distributed storage units. The tool will be responsible for sending the final solution directly to the controllers of the LV storage. (iii) Schedule emergency control actions: In case the MG does not have enough power and/or energy reserve capacity to guarantee the power balance, it will be necessary to exploit the flexibility available at the HEMS to ensure power balance and thus avoid the system collapse.

\section{$7 \quad$ Validation and next steps}

To evaluate the effectiveness of the MG emergency balance algorithm, a LV network with four storage devices was considered (a $30 \mathrm{~kW} / \mathrm{kWh}$ VSI and two $10 \mathrm{~kW} / \mathrm{kWh}$ PQ controlled). It is assumed that some LV clients have installed DER such as PV panels and small storage units.

An illustrative time horizon of $1 \mathrm{~h}$ was considered, corresponding to the maximum time the MG was initially planned to operate islanding. The time horizon was divided in four time steps of $15 \mathrm{~min}$. Table 1 presents the load and microgeneration forecasting.

As observed in Table 1, MG load exceeds generation, meaning that in the VSI there is an excess of load compared to

Table 1 Operation scenario considered for the test system

\begin{tabular}{lcccc}
\hline Time interval & 1 & 2 & 3 & 4 \\
\hline load, kW & 32 & 33 & 37 & 45 \\
microgeneration, kW & 14 & 22 & 12 & 10 \\
MG power balance, $\mathrm{kW}$ & -18 & -11 & -25 & -35 \\
\hline
\end{tabular}

microgeneration. The storage units participating in the dispatch are identified as PQ from this point forward. In the moment subsequent to the MG islanding, the following SoC of the MG storage devices are, respectively: $V S I=30 \%, P Q 1=90 \%$, $P Q 2=80 \%$ and $P Q 3=10 \%$. To demonstrate the effectiveness of the proposed algorithm, it was also simulated a case of comparison where the dispatch of microgeneration is done based only in the power limitations. Let us consider that the comparison case it's scenario 1 and the proposed algorithm it's scenario 2 . The main results are depicted in Figs. 8-10.

As observed in Fig. 8, PQ 3 will fully discharge at the end of first period, having the MG only two PQ storage units supplying the power unbalance between load and MS generation during the remaining periods. In contrast, as observed in Fig. 9, the algorithm
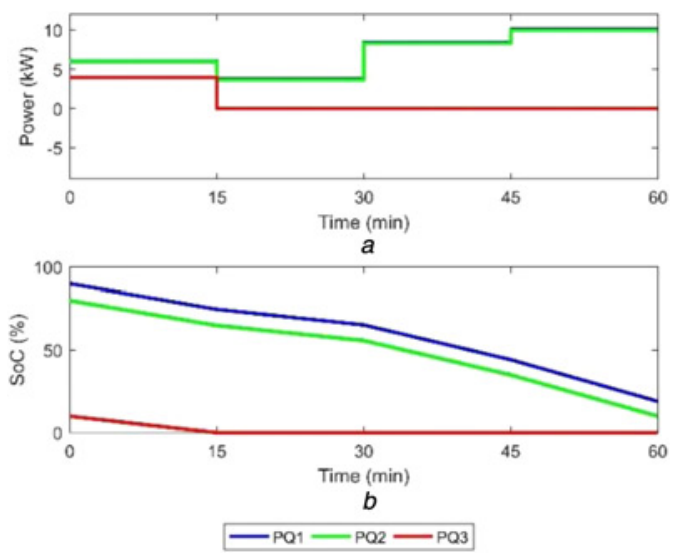

Fig. 8 Scenario $1-P Q$ unit's operational strategy

(a) Active power set-points, (b) State-of-charge
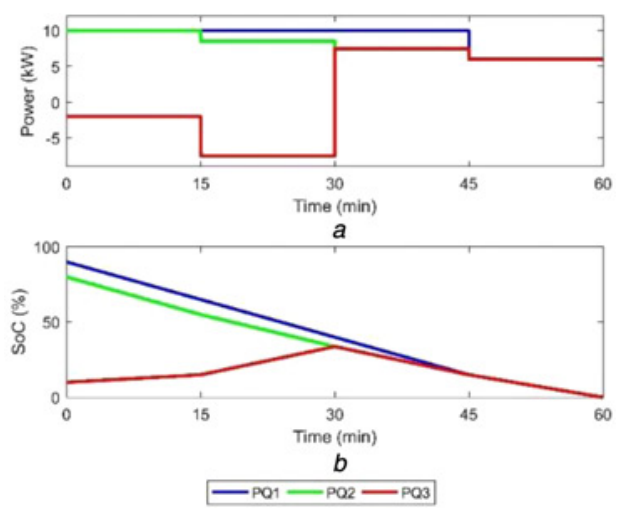

Fig. 9 Scenario $2-P Q$ unit's operational strategy

(a) Active power set-points, (b) State-of-charge
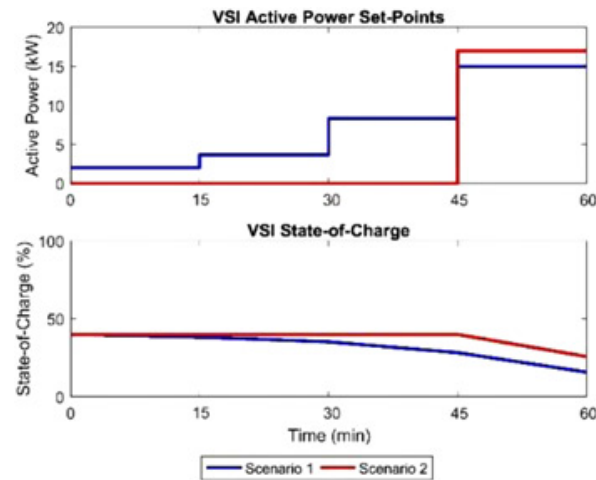

Fig. 10 VSI operational strategy 
will take advantage of the load and microgeneration forecasts, leading the PQ 3 to charge in the first two periods, allowing it to reduce the unbalance between load and MS generation presented in periods three and four.

The impact of the proposed dispatch strategy in the VSI is presented in Fig. 10. Unlike scenario 1, the VSI storage unit in scenario 2 will not exhibit such an extreme discharge due to the contribution of all PQ storage devices in the MG power balance.

Before the implementation in Évora demonstrator, the MG emergency balance tool will be extensively tested in INESC TEC laboratory facilities as well as in Labelec facilities. The laboratory facilities allow to build a small-scale LV test system incorporating grid and residential DER and loads. In addition to the electric infrastructure, the laboratory includes the SM infrastructure implemented in Portugal.

\section{Acknowledgments}

This work was carried out as a part of the SENSIBLE project (Storage ENabled SustaInable energy for BuiLdings and communitiEs - www.h2020-projectsensible.eu) that has received funding from the European Union's Horizon 2020 research and innovation programme under grant agreement No 645963.

\section{References}

1 EDP Distribuição: 'LV secondary switchgear technical specifications'

2 Filipe, N.: 'Protection system analysis in LV Grid, with high DG penetration, in Parallel and Islanding Operation'. CIRED, 2017

3 André, R.: 'EDP Distribuição inovgrid first electrical energy storage project'. Cired, 2015

4 André, R.: 'Energy services bridging the gap between residential flexibility and energy markets'. Cired, 2017 